a

CENTROALGORITMI

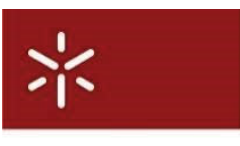

Universidade do Minho

Luiz Alberto Lisboa da Silva Cardoso, João Luiz Afonso, Miguel Comesaña Martinez, Andrés Augusto Nogueiras Meléndez

"RFID-Triggered Power Activation for Smart Dynamic Inductive Wireless Power Transfer"

IEEE IECON Industrial Electronics Conference, pp. 6967-6973, Beijing China, Oct. 2017.

https://ieeexplore.ieee.org/document/8217218

DOI: 10.1109/IECON.2017.8217218

ISBN: 978-1-5386-1127-2

This material is posted here with permission of the IEEE. Such permission of the IEEE does not in any way imply IEEE endorsement of any of Group of Energy and Power Electronics, University of Minho, products or services. Internal or personal use of this material is permitted. However, permission to reprint/republish this material for advertising or promotional purposes or for creating new collective works for resale or redistribution must be obtained from the IEEE by writing to pubs-permissions@ieee.org. By choosing to view this document, you agree to all provisions of the copyright laws protecting it.

(C) 2017 IEEE 


\section{RFID-Triggered Power Activation for Smart Dynamic Inductive Wireless Power Transfer}

\author{
L. A. Lisboa Cardoso, J. L. Afonso \\ Centro ALGORITMI, Department of Industrial Electronics, \\ University of Minho, Campus de Azurém, \\ 4800-058 Guimarães, Portugal
}

\author{
M. Comesaña Martinez, A. A. Nogueiras Meléndez \\ Department of Electronics Technology, University of Vigo, \\ Vigo, Pontevedra 36310, Spain
}

\begin{abstract}
A RFID-triggered power activation system is devised, implemented and tested as the key sensing mechanism for controlling wireless power transfer from an inductive lane to a moving lightweight electric vehicle. The RFID reader and tag used are based on ISO/IEC-14443A compatible types, working at $13.56 \mathrm{MHz}$, one of the least expensive currently available passive near-field technologies. In order to fit lightweight electric mobility application requirements, the RFID magnetic antennas are redesigned to larger than standard sizes, both at reader and tag sides, increasing the detection volume without increasing the required reading power, thus permitting the reuse of commercial RFID circuits and embedded protocol software. To trigger the wireless power transfer, a RFID reader coil is placed magnetically orthogonal to each primary power coil to be controlled, at the extremity where electric vehicles are expected to come from, in a one-way traffic. The power is turned off automatically when the vehicle leaves the primary coil field and the power demand is extinguished, or when time-out is reached. An inductive lane constituted of a sequence of such RFID-controlled primary coils can detect the presence of authorized electric vehicles and smartly energize the lane, manipulating the unique identifier codes and other information stored in the on-board tag to more efficiently handle dissimilar power level demands, and to implement tolling. The performance of the system is predicted based on the reconstructed 3D digital model of the RFID main detection lobe.
\end{abstract}

Keywords - Inductive wireless power transfer; smart electric power control; ISO/IEC-14443A RFID applications.

\section{INTRODUCTION}

Dynamic inductive wireless power transfer (DIWPT) is a theme of current investigation for road electrification, with many recent technical achievements and prototype systems implemented [1]. These systems basically consist in embedding primary coils underneath the vehicle's path and energizing them in sequence, with $\mathrm{AC}$ currents, at the right timing, every time a vehicle with an on-board secondary coil is presented over each primary coil. Under resonant circuit conditions and using adequately low-loss wiring, the primary and secondary coils form an air-core transformer that allows energy to flow from lane to vehicle at practical overall efficiencies, in the expected order of 70 to $80 \%$ [2]. Fig. 1 shows a simplified system architecture representation of such a system. As the vehicle moves forward along its path, its pick-up (secondary) coil will sequentially cross the magnetic field generated by the stationary primary coils placed underneath vehicle's path floor surface. For safety and efficiency reasons, only the primary coils in position of significant mutual induction with secondary coils should be activated at a given time. The system effectiveness depends not only on good magnetic coupling and power electronic design, but also on the appropriated time-spatial coil activation pattern.

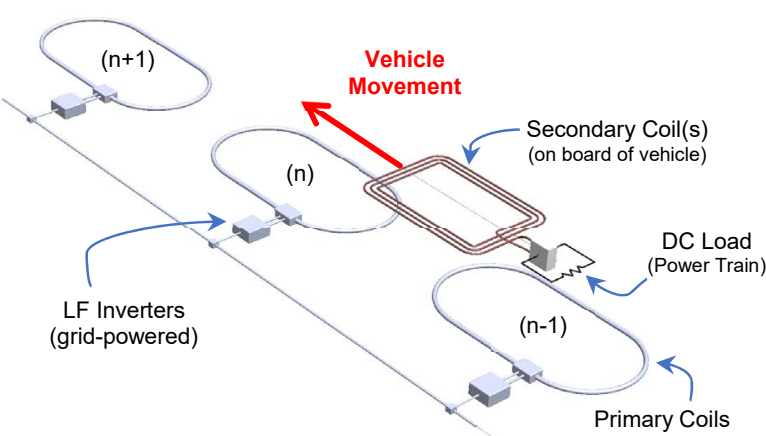

Fig. 1. Schematic representation of a typical dynamic inductive wireless power transfer configuration.

Considering the vehicle cooperative behavior, the detections schemas maybe either passive as in [3] or active, draining some vehicle on-board power, as in [4].

RFID (Radio Frequency Identification) systems have been used before for wirelessly powering and reading low power sensors integrated to tags [5]. This work, however, prospects the utilization of standard near-field RFID, with both reader and tag antennas modified, as a strategy to detect primary to secondary coil alignments and activate other specialized wireless power transfer circuitry. A design was conceived targeting lightweight electric vehicles, such as pedal electric cycles (PEDELEC), also referred as electrically power assisted cycles (EPAC) [6], where vehicles' nominal power is limited to $250 \mathrm{~W}$, with possible peaks of $400 \mathrm{~W}$, and the maximum vehicles' speeds range from 25 to $35 \mathrm{~km} / \mathrm{h}$, depending on local legislation [7]. The principles and the analysis method can be, however, extended to higher speed electric vehicles and inductive lanes.

\section{RFID ACTIVATED INDUCTIVE POWER TRANSFER}

Early lab experiments with DIWPT used photocell interruption to sense vehicle's secondary coil to lane's primary coil alignment, and sequentially power them on [3]. One passive 
sensing strategy has been recently proposed by Kamineni et al., at the University of Auckland [8], which is based on the magnetic coupling enhancement between two sequential primary coils when a third pick-up coil, the vehicle's on-board secondary coil, is travelling in between them. This strategy was demonstrated to work with double " $D$ " primary coils [9] but could expectably be adapted to any other primary coil configuration, provided that these coils are closely enough spaced along the vehicle's path. The sole use of this technique, however, should not permit the initial vehicle detection and identification at the first primary coil in a row.

RFID, on the other hand, have long been used on board of vehicles for both road tolling and access control. The inductive power transfer (IPT) concept is implicit in passive RFID.

\section{A. RFID Subsystem Requirements for IPT Activation}

(R.1) Guaranteed speed of detection and identification. For a tag to be detected and read, it must remain continuously within the region of the space where detection can occur, the so called the detection volume. The guaranteed speed can then be estimated by the ratio between the shortest travel distance of the tag, while crossing the detection volume, and the maximum time required to successfully process a tag, detecting and identifying it. For the e-bike study case considered, the value was established at $25 \mathrm{~km} / \mathrm{h}$.

(R.2) Detection distance. Both the RFID reader and primary power coils are stationary, while the tag and the secondary coil are installed on board of the vehicle, at fixed relative position, it is convenient that the maximum detection distance be slightly larger than the IPT inter-coil distance, which was designed at $13.5 \mathrm{~cm}$ for the e-bike lane.

(R.3) Lateral displacement tolerance. The RFID reader should be capable of correctly detecting and identifying the tag, even if laterally misplaced up to a certain width embracing the center detection line. The RFID detection center line does not necessarily coincide with the center of the lane. But it is expected to be parallel to both, which usually shall coincide. The lateral displacement tolerance of the RFID should be equal to or slightly greater than the lateral displacement tolerance of the secondary coil with respect to the primary coil, arbitrated at $39 \mathrm{~cm}$, as justified in Table III.

(R.4) Decoupling from magnetic power circuit. The RFID subsystem is assumed to perform its task of detecting tags and issuing a triggering signal to activate the power inverter before the primary coil is energized. So, functional sensitiveness to the power transfer magnetic field is not critical. However, both the reader and the tag must at least withstand the voltages possibly inducted in their coils during the energization periods.

\section{B. Selection and Characterization of RFID Technology}

The correct recognition of codes retrieved from RFID tags as the key to activate devices and systems is the typical application for this technology. Today's existing standards use the frequency band of $\mathrm{LF}(125 \mathrm{kHz}$ or $134.2 \mathrm{kHz}), \mathrm{HF}$ (13.56 MHz), UHF $(860 \mathrm{MHz}-960 \mathrm{MHz})$ or SHF $(2.45 \mathrm{GHz})$. When selecting a RFID technology for DIWPT, the following considerations should be observed:
- UHF and higher frequency RFID systems operate in farfield mode (irradiative), with high data bit rates and fast response, favoring the processing of faster vehicles. They are already used in applications involving detection moving vehicles. However, their detection distances are usually much greater than the typical airgap in IPT configurations, what may lead to a too large anticipation in turning the corresponding primary coil on, if used as a sole method of detection. These higher frequency RFIDs are also more sensitive to propagation conditions and potential interferences from other tags in nearby vehicles. That makes it difficult to predict the correct time to turn each power coil in the lane on, based on the detection signal coming from the RFID reader unit.

- HF and lower frequency RFID systems, operating in near-field (inductive) mode may have reading distances of up to $1 \mathrm{~m}$ (see Table I). These distances are compatible with inter-coil distances in current vehicular IPT applications. The field strength from the primary power coil, however, tends to affect the operation of the RFID: In a first level, by interfering in the modulation process that permits communication between the RFID reader and the tag. Further, if the IPT field is too strong, induced voltages, in tag or reader coils, can damage the RFID circuits, in spite of the frequency separation of power and RFID signals. Among these systems, higher frequency band correlate with higher bit rates and lower response times, what favors the lane power response time and the higher vehicles' speed.

This initial screening processes pointed out the $13.56 \mathrm{MHz}$ as the most a priori promising RFID band. Two commonly found non-proprietary standards at $13.56 \mathrm{MHz}$ were compared, the ISO/IEC-14443 (with variants A and B) and the ISO/IEC15693. Their main performance parameters are summarized in Table I. While ISO/IEC-15693 provides reading distances that better complies with requirements R.2 and R.3, the ISO/IEC14443 standards have faster bit rates. Decision was towards initial experimentation with the ISO/IEC-14443A, due to its higher availability and lower cost.

\section{TABLE I. NEAR-FIELD RFID STANDARDS AT $13.56 \mathrm{MHz}$}

\begin{tabular}{|c|c|c|}
\hline Performance Parameter & $\begin{array}{c}\text { ISO/IEC } \\
\mathbf{1 4 4 4 3 \boldsymbol { A } / \boldsymbol { B }}\end{array}$ & $\begin{array}{c}\text { ISO/IEC } \\
\mathbf{1 5 6 9 3}\end{array}$ \\
\hline \hline $\begin{array}{c}\text { Magnitude of } \\
\text { Maximum Reading } \\
\text { Distance (m) }\end{array}$ & $\begin{array}{c}0.1 \\
\text { ("Proximity") }\end{array}$ & $\begin{array}{c}1 \\
\text { ("Vicinity") }\end{array}$ \\
\hline $\begin{array}{c}\text { Maximum Data Rates } \\
\text { (kbit/s) }\end{array}$ & $\begin{array}{c}\text { A: } 105.9 \\
\text { B: } 847.5\end{array}$ & 26.4 \\
\hline $\begin{array}{c}\text { Required Field } \\
\text { Strength (A/m) }\end{array}$ & 1.5 to 7.5 & 0.15 to 5 \\
\hline Anti-collision Protocol & Yes & Yes \\
\hline
\end{tabular}

The RFID 14443 standards [10] [11] [12] refers to the RFID reader as the proximity coupling device (PCD) and the RFID tag as the proximity integrated circuit card (PICC). The ISO/IEC14443A-compatible tag chip used in the experiment is a product marketed by the NXP Semiconductors, under the name MIFARE Classic [13], commonly used in public transportation, car parks and electronic toll collection cards worldwide. 
The dimensions for MIFARE PICC antenna are standardized [10], limited to a fixed contour inside an ISO/IEC-7810:2003 card, which is $85.60 \mathrm{~mm} \times 53.98 \mathrm{~mm}$, while the PCD antenna dimensions are not size constrained. The dimensions of the printed circuit board (PCB) antenna used in the characterization were $38 \mathrm{~mm} \times 42 \mathrm{~mm}$, typical in commercial card readers.

Experimental plots of detections contours, taken on plans that are parallel to the reader coil, at different heights, are shown Fig. 2a. Due to the small dimensions of the reader PCB and the presence of a connector and other electronic components on its surface, some mechanical restriction to measure points close to the reading coil $(z=0)$ was observed, and these were extrapolated by presumed symmetry and continuity, generating the curves drawn in blue. The black and red plots on the contours were individually measured by displacing the card on the plane.
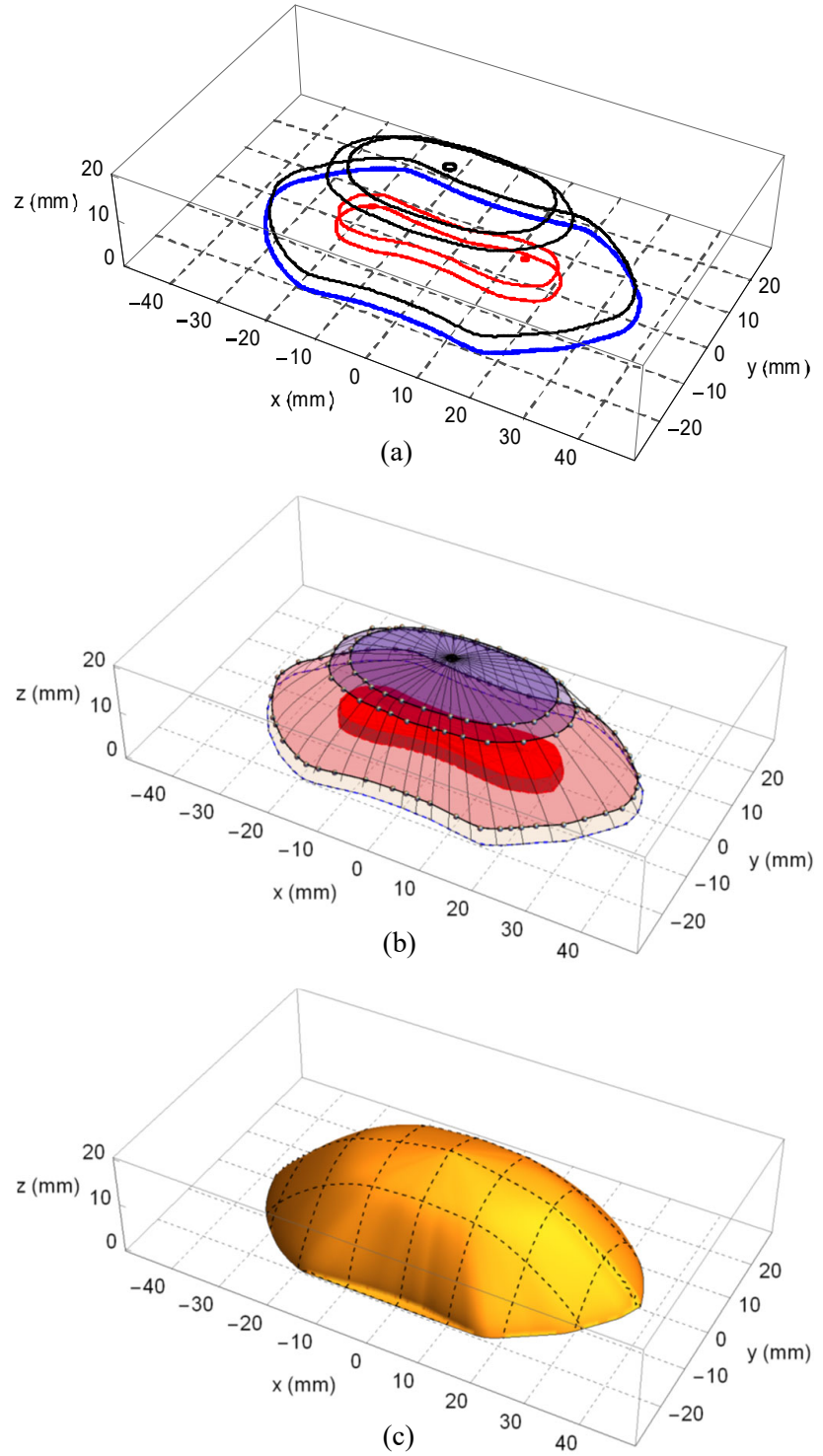

Fig. 2. Major detection lobe of a standard ISO/IEC-14443A reader-tag antenna pair, as reconstructed from measurements at different heights above RFID coil plane.
The red contour indicates a central exclusion bone-shaped region, about $2 \mathrm{~mm}$ high, where, due to larger size of the tag over the reader coil, no tag detection in observed.

The plots were digitalized and used to create a 3D model of the detection volume for the reader/tag pair, as show in Fig. $2 b$ and Fig. 2. The maximum detection height was found to be $20 \mathrm{~mm}$, with the base of the detection volume inscribed in a rectangle of roughly $75 \mathrm{~mm}$ x $40 \mathrm{~mm}$.

\section{Extending Operation Range of RFID $14443 \mathrm{~A}$}

ISO/IEC 14443A-based RFID systems can have the operation range extended up to of $10 \mathrm{~cm}$ from the RFID reader, depending on the reader coil dimensions. The actual operation range of commercial systems, however, as measured and documented in section II-B, usually lay in the 2 to $5 \mathrm{~cm}$ range. One method of extending the reading range of ISO/IEC$14443 \mathrm{~A}$, further than that, is to amplify reading currents and reading antenna dimensions [14]. If this is done in conjunction with the use a battery powered tag that actively emulates load modulation, ranges of a few meters are possible [15].

In DIWPT applications the RFID readers are expected to be permanently scanning for vehicles, to activate power at vehicle/tag presentation: Therefore, increasing the reading current level would result in an inconvenient higher lane standby power, reducing overall electrical efficiency. Also, the need to replicate these upgraded reading circuits along the lane would make the system more expensive. Battery operated tags on the other hand, while very effective in extending the detection range, require an auxiliary battery on board of vehicle, to guarantee the energy harvest can be started. The option adopted in this work was, however, to first test the limits of the achievable range extension just by enlarging the reader and tag antenna coils, using no signal amplification and keeping the tag purely passive.

The strategy for enlarging the reader antenna without changing its circuit parameters was to simultaneously reduce the number of coil turns and increase the $a \times b$ rectangular coil dimensions. The maximum antenna size was then obtained with a single coil turn, $50 \mathrm{~cm} \times 29 \mathrm{~cm}$, exhibiting the same inductance of the original reader coil that consisted a of fourturn rectangular spiral coil laid on printed-circuit board, with external dimensions of $38 \mathrm{~mm} \times 42 \mathrm{~mm}$. Larger reader antennas could be obtained exhibiting the same initial inductance, by using thicker wirer and less turns. However, for near-field operation the antenna perimeter, $2 a+2 b$, should be kept as low as possible, below the near-field mode limit dimension given by:

$$
2 a+2 b \ll \frac{\lambda}{2 \pi}=\frac{22.1}{2 \pi} \cong 3.5 \mathrm{~m}
$$

A practical and more conservative limit value, often adopted as a recommendation, is $\lambda / 10$ instead, numerically equivalent to $2.21 \mathrm{~m}$ at $13.56 \mathrm{MHz}$. In this way, the newly designed reader coil, with $a=50 \mathrm{~cm}$ and $b=29 \mathrm{~cm}$, has a perimeter of $1.58 \mathrm{~m}$, within $a \cong 30 \%$ margin away from the $\lambda / 10$ maximum recommended length. Once near-field operation is presumed, neglectable irradiation losses are observed, and the reader and the tag behave as electric circuits that are coupled by an air-core transformer. 
The new larger reader coil has made to exhibit the same electric circuit parameters as the original antenna, so it can be driven by the same original PCD circuit. But it will generate a weaker field strength. To compensate for that, the tag coil is also modified, with an increase in the area of its cross-section and number of turns, using also a thicker conductor. A balanced design is made so that the inductance of the original tag coil is also matched. The new tag coil is then connected to the tag chip though a compensating network and new reader/tag pair exhibits an extended range of operation.

The same procedure measure the contours of limit $100 \%$ detection, previously used in the characterization of the MIFARE standard card, was used to characterize the new reader/tag coil pair, generating the curves plotted in Fig. 3a, and the 3D model in Fig. 3b, a visibly larger volume than that of the original MIFARE card, shown in Fig. $2 b$, but conveniently not presenting any detection-blind zone inside the main lobe.

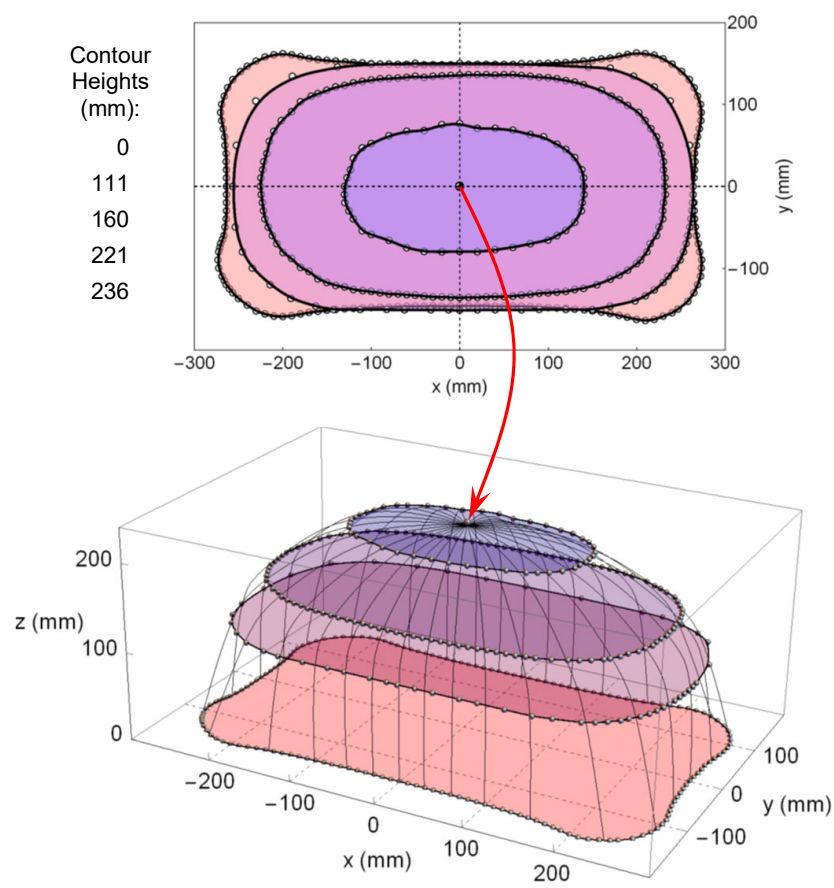

Fig. 3. Main detection lobe for the modified ISO 14443A reader-tag antenna pair, as reconstructed from measurements.

The new envelope dimensions of the reading volume are shown in Table II. The result is an increase in the detection range from $20 \mathrm{~mm}$ (as extracted from the model inFig. 3) to $236 \mathrm{~mm}$, that is, a linear gain in range of approximately 12 times, if compared with the detection range of the original MIFARE card-reader pair.

Beyond obtaining better range detection and lateral tolerance alignment parameters with the new reader/tag pair, the total detection volume is also increased: Thus, the transit time of the tag when crossing the detection space of the main detection lobe of the PCD antenna is also increased, at a given tag/vehicle speed. Or conversely, for a fixed given identification rate to be performed by the PCD, more tag/vehicle speed can be handled.
TABLE II. DETECTION CONTOURS FOR RE-DESIGNED RFID ANTENNAS

\begin{tabular}{|c|c|c|c|}
\hline \multicolumn{5}{|c|}{$\begin{array}{c}\text { Principal Dimensions of Detection Contours } \\
\text { for 100\% RFID Detection Rate }\end{array}$} \\
\hline Height (mm) & $\begin{array}{c}\text { Transversal } \\
\text { Width (mm) }\end{array}$ & $\begin{array}{c}\text { Longitudinal } \\
\text { Depth (mm) }\end{array}$ & $\begin{array}{c}\text { Cross-Section } \\
\text { Area }\left(\text { cm }^{2}\right)\end{array}$ \\
\hline \hline 0.0 & 527 & 295 & 1606 \\
\hline 111 & 521 & 301 & 1423 \\
\hline 160 & 457 & 272 & 1068 \\
\hline 221 & 270 & 155 & 345 \\
\hline 236 & 0 & 0 & 0 \\
\hline
\end{tabular}

\section{Performance ANALysis}

Based on the major lobe digital model of the reading volume, reconstituted from measurements by numerical interpolation, programmed in Wolfram Mathematica software tool, it is possible to anticipate important system behavior characteristics, such as misalignment tolerances and maximum vehicle speed for assured detection and correct radio-frequency identification. With the aid of the model, is also possible to select the best possible height to install the tag on the vehicle.

\section{A. Lateral Alignment Tolerance}

The power transfer capacities of the target DIWPT system, an experimental inductive lane for e-bikes [7], are given at different misalignment conditions, in Table III.

TABLE III. POWER TRANSFER CAPACITIES AND CORRESPONDING VEHICLE ALIGNMENT TOLERANCES

\begin{tabular}{|c|c|c|}
\hline $\begin{array}{c}\text { EV Power } \\
\text { Demand Level }\end{array}$ & $\begin{array}{c}\text { Power } \\
(\mathbf{W})\end{array}$ & $\begin{array}{c}\text { EV to Lane Maximum } \\
\text { Misalignment Width, w (cm) }\end{array}$ \\
\hline \hline Peak & 400 & 20 \\
\hline Nominal & 250 & 27 \\
\hline$-3 \mathrm{~dB}$ & 200 & 29 \\
\hline Low & 100 & 39 \\
\hline
\end{tabular}

Considering $100 \mathrm{~W}$ as the minimum level of useful assistance power ("Low" level, in Table III), RFID correct operation must be verified at corresponding alignment tolerance, that is, vehicle detection must be assured for any $w \leq 39 \mathrm{~cm}$. For a given height, $h$, this width, $w$, can be directly checked by inspecting the cross-section of the major detection lobe at the height $h$, as illustrated in Fig. 4. If a rectangle of width $w$ is inscribed in the cross-section perimeter, its other side, $d$, will be the minimally assured depth of crossing, for all lateral displacements $\pm x$ such that $|2 x| \leq w$.

By examining the contour plot in Fig. 4 , it is possible to infer that, using a rectangular reader coil, except possibly at very low tag heights, which are not practical, the maximum tolerance alignment will decrease with height, until the cross-section collapses to a point at $h=236 \mathrm{~mm}$, the maximum possible tag height, above which there will be no tag detection. 


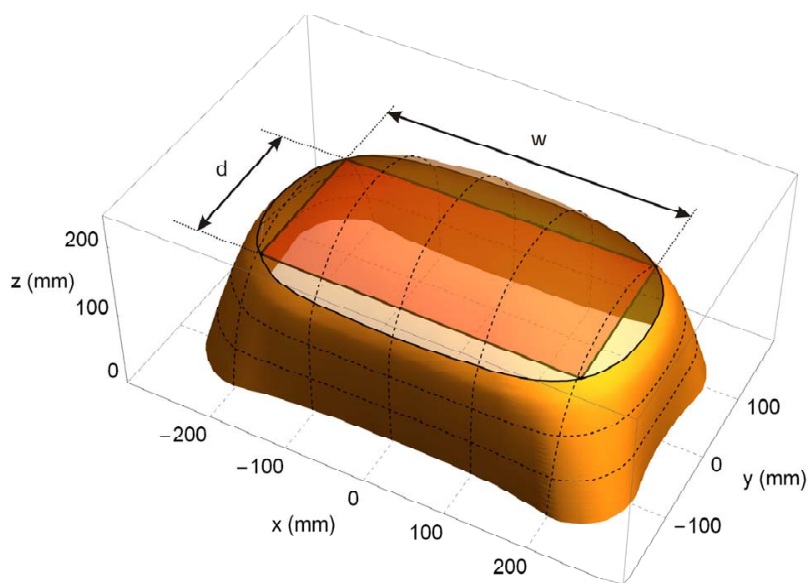

Fig. 4. Numerically computed horizontal cross-section of the main RFID detection lobe for the modified reader-tag antenna pair.

\section{B. Maximum Vehicle Crossing Speed for Detection}

The amplitude modulation (ASK) used in ISO/IEC-14443A is not affected by vehicle's speed, provided the tag coil is kept within the reader detection volume and parallel to reader coil, often a reasonable assumption in vehicular applications. In this manner, to assure detection is sufficient for the tag to remain inside the detection volume enough time for the exchange of the defined messages in the RFID protocol, then to process the tag UID data and decide whether the power activation triggering signal should be issued. In laboratory experiments, this time, $\Delta t_{p}$, was consistently measured to be limited to 32 milliseconds, working with an Arduino module based on the NXP MFRC522 reader/writer IC [16] and software from Arduino RFID Library for MFRC522, with minor modifications, for measuring the duration of the identification cycles. The code was run in a ATmega328P processor (Arduino UNO R3).

For a vehicle/tag assembly to be detected, its transit time along the main lobe cross-section, at height $h$ and lateral misalignment $x$, should be greater or equal to $\Delta t_{p}$. Then its average speed crossing the detection volume at any displacement within the width $w$, should be given by (2) and (3):

$$
\begin{gathered}
v(x) \leq v_{\max }(x)=\frac{d(x)}{\Delta t_{p}} \leq \frac{d_{\min }}{\Delta t_{p}} \\
v_{\max }=\frac{d_{\min }(h, w)}{\Delta t_{p}}
\end{gathered}
$$

This maximum speed can be numerically computed from the reconstructed digital model of the main detection lobe, for given values of $h$ and $w$. At the limit case whereas $w=0$, i.e., perfect vehicle alignment, the relationship between tag the height and the maximum vehicle speed is given by the black dashed curve in Fig. 5. The other curves in Fig. 5 show how the maximum speed decays, as the required alignment tolerances, $w$, are increased. The curves are homothetic to intersections of the detection lobe in Fig. 4 with vertical parallel planes at $x=w / 2$.

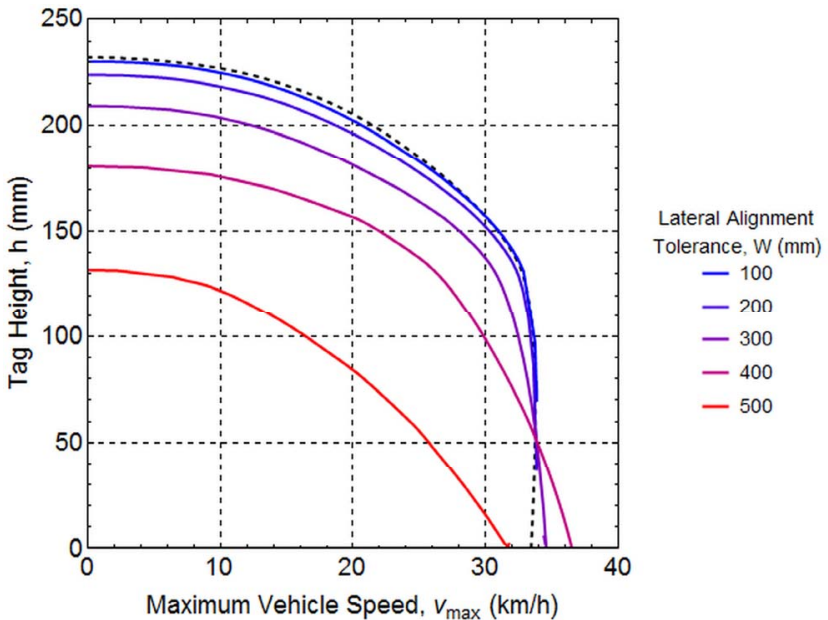

Fig. 5. Predicted maximum electric vehicle speed for guaranteed detection, as a function of the height of the tag above the RFID reader antenna and the desired lateral alignment tolerance.

In practice, tag heights less than the power coils "air-gap" (which is greater than the vehicle-to-ground clearance distance) are not mechanically allowed, and the curves in Fig. 5 should only be considered relevant above that quota, in the case of the presently studied system, $135 \mathrm{~mm}$.

The use of MIFARE ISO/IEC-14443A RFID with modified antennas was then verified to be compatible with the requirements of lateral tolerance widths of $40 \mathrm{~cm}$ and vehicle speeds up to $25 \mathrm{~km} / \mathrm{h}$, being adequate to be used in conjunction with the prototype e-bike inductive lane tested. Measurements with an instrumented moving vehicle are yet due to be realized, as an ultimate design check for maximum achievable speed.

While this analysis methodology holds in other applications, the impossibility of significantly further increasing the coil size, without leaving the desired near-field operation, would likely prevent its application at speeds higher than $45 \mathrm{~km} / \mathrm{h}$ - a number that results if the reader coil is rotated $\pi / 2$ with respect to direction of movement. This would be obtained, however, at the expense of a tighter alignment tolerance, in the order of $25 \mathrm{~cm}$.

\section{RFID SYSTEM INTEGRATION}

Each of the RFID coils must be installed close to the corresponding power coil, the reader coil on the primary side, and the tag coil on the secondary (R.4, in section II.A). The collocation of the coils has, as its main directive, to preserve the integrity of the RFID circuits upon energization of the power coils. This is obtained if the RFID and power coils are installed at magnetically orthogonal relative positions, both on the primary (lane) side and on the secondary (vehicle) side. This is a special condition where the total magnetic flux from one coil to another is zero, due to positive and negative fluxes cancelling each other, as illustrated in Fig. 6. Since they have to be parallel, almost in the same plane, due to construction constraints, the coils will be placed at eccentric positions. 


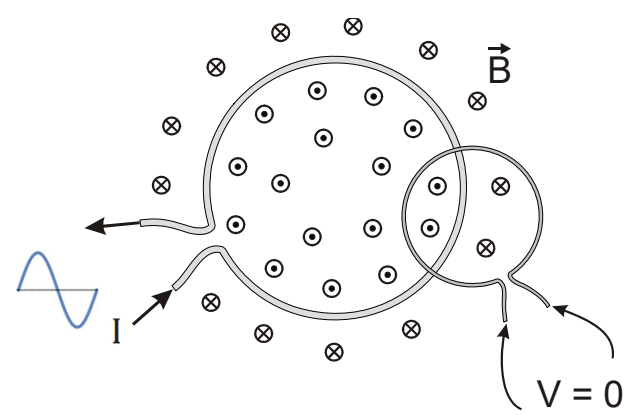

Fig. 6. Example of coils in magnetically orthogonal relative position.

\section{A. RFID Tag and Secondary Power Coil Integration}

The vehicle used to test the concept is an electric bike, with the secondary power coil wound around the rear wheel, what results in difficulties to position the tag simultaneously aligned with the longitudinal center axis and close to the secondary power coil. The redesigned tag with a newly built antenna, consisting of 6 cylindrical-helix turns of $1.5 \mathrm{~mm}^{2}$ cross-section copper wire, $128 \mathrm{~mm}$ of diameter and $18 \mathrm{~mm}$ height, is then placed on a lateral side of the secondary coil, as shown in Fig. 7. When the bike moves forward, the center of the tag coil displaces along $s_{2}$, parallel to bike center axis $s_{0}$, at a distance $d_{\text {tag }}$ from $s_{0}$, which is experimentally established as to create the magnetic orthogonality condition between coils. The passive tag circuitry then stays electrically decoupled from the secondary power circuitry. The exact position of the tag coil along $S_{2}$ is determined after the reader coil is positioned in the primary, as explained in section IV.B.

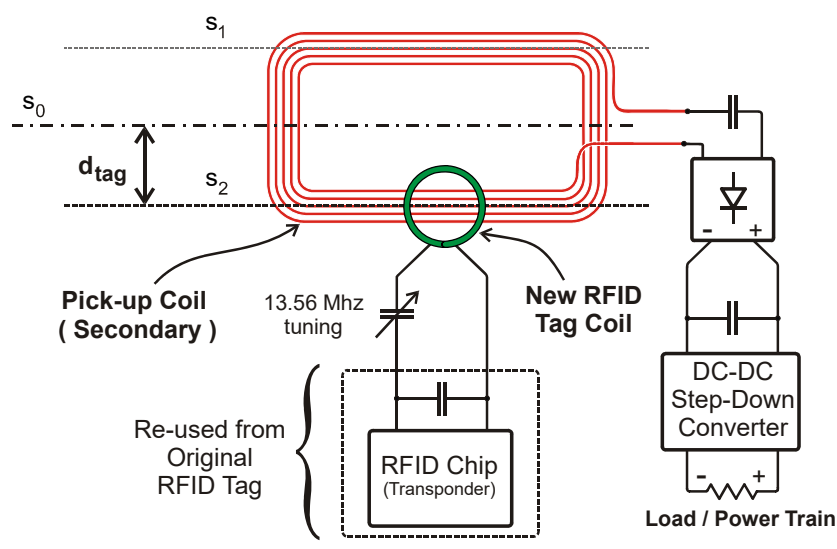

Fig. 7. RFID tag integration on board of electric vehicle.

\section{B. RFID Reader Installation on Lane}

The RFID reader coil, a single turn $50 \mathrm{~cm} \times 29 \mathrm{~cm}$ rectangular one, made with double $0.5 \mathrm{~mm}^{2}$ cross-section wire, is then installed on one of the extremities of the primary coil, parallel to it, but eccentric to the coil (and lane) longitudinal axis by the same distance $d_{t a g}$ established in the secondary integration. This causes the reader coil to be aligned to the tag when the primary coil is center aligned to the secondary coil. The exact position of the center of the reader coil is obtained by intersecting line $r_{2}$ with the zero-mutual inductance locus, the red curve in Fig. 8, experimentally determined. At this point, the primary power coil and the RFID reader coil will be decoupled, except by a capacitive coupling due to parasite capacitances, which were verified to be irrelevant to RFID operation.

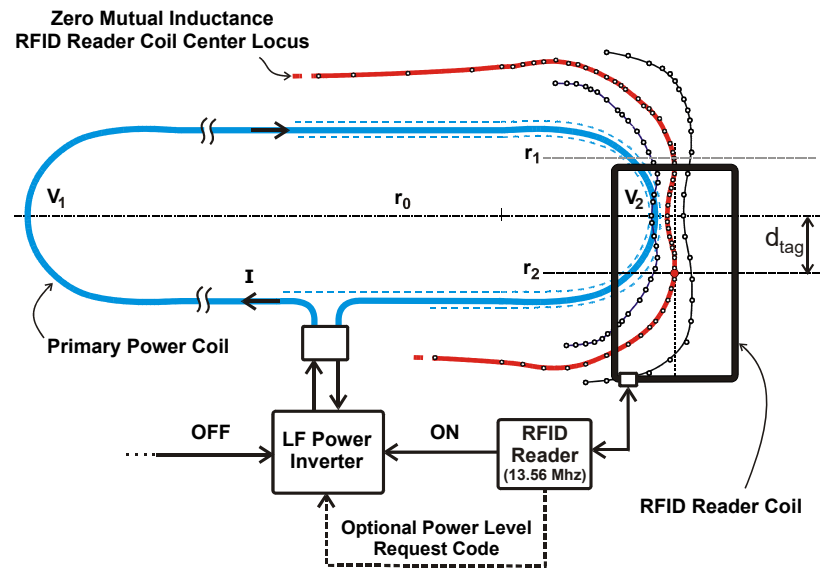

Fig. 8. Integration on the lane, with the RFID reader coil positioned at the extremity of the primary coil the vehicle is expected to come from.

The exact location of the tag coil in the secondary is then fixed by positioning the vehicle over the lane assembly and requiring that the center of RFID coils coincide at the point where energy transfer must be triggered, that is, a point at the approaching route of the vehicle where sufficient coupling between primary and secondary power coils start to be observed, and thus, the power inverter can be activated. After activation, the currents in primary and secondary coils can affect normal RFID operation, but not damage it. The RFID status, however, becomes irrelevant after power is activated.

The RFID reader reads a unique identifier (UID) code, transponded by the tag, which can be 4 or 7 bytes long, depending on the MIFARE card model. The reader processor then criticizes it and issues an activation signal that triggers the power inverter on. In the current prototype used for testing the concept, the UID of four bytes is merely checked to be in a predefined list of enabled users. In real applications, other more complex algorithms and on-line database checking can be engineered, provided a fast enough response is guaranteed. Beyond the UID, other values stored in the registers of the tag chip can be manipulated at toll-stops (longer transaction times), for instance, to decrement the user "credit", in a similar manner the ISO/IEC-14443A MIFARE cards are used in current toll applications.

\section{CONClusion ANd Future WORK}

The reuse of standard near-field operation ISO/IEC-14443A RFID circuitry and software was proposed as a means of triggering dynamic inductive wireless power transfer to electric vehicles. This scheme not only can detect approaching electric vehicles, but also extract vehicles' authorization, tolling and power demand level codes, enabling more efficient electrification of an inductive lane. If lane segments are adequately limited in length, no two vehicles can be over the same segment, and the entire lane can simultaneously and more 
efficiently handle vehicles with dissimilar power requirements. If the vehicle power train control is to be integrated with the tag, the same vehicle could present different power demand codes to be transponded to the lane, at different times.

Trial experiments were conducted using a NXP MIFARE commercial family of RFID products, which is ISO/IEC$14443 \mathrm{~A}$ compatible and operates at $13.56 \mathrm{MHz}$. Actual measurements of the major lobe for a newly designed reader-tag antenna pair indicate a maximum vehicle speed, for guaranteed detection and successful DIWPT operation, in the range of $25 \mathrm{~km} / \mathrm{h}$ to $30 \mathrm{~km} / \mathrm{h}$, at a lateral alignment tolerance of $40 \mathrm{~cm}$. Further testing is due with the vehicle instrumented, as an ultimate proof of these predicted figures. While this speed is not high enough to address traffic in highways, it fits satisfactorily slower moving lightweight electric vehicle applications, such as electrically power assisted cycles and industrial applications using similarly speed-rated electric vehicles.

Although one-way traffic is required in the configuration tested, the present results could be extended for nonsimultaneous two-way traffic as well, by using one RFID reader at each extremity of the controlled primary coils. To minimize the complexity and cost added in a larger lane system, this method can be used as a strategy for identifying the vehicle and activating the lane power start, by controlling the first coil only in a row of coils, since other simpler methods to control the coils following the first can be found, if the coils are close enough.

The RFID have some known security issues that are being researched, and improvements are constantly made to this technology. The risk of tag cloning and eavesdropping attacks may however require detailed analysis before the use this technology in high-end wireless power transfer and associated tolling applications. For low-end, low cost applications of IPT, such as e-bikes and similar lightweight electric vehicles, the use of RFID technology for activation and tolling is expected to offer an acceptable trade-off between security and functionality.

In this first implementation, the RFID software was not optimized for timing. The RFID standard considers the possible existence of multiple simultaneous tags in the detection volume and the firmware implements algorithms to handle the potential collision of messages from the different tags. In the vehicular application described, however, it is unlikely that two or more vehicles can coincide over the extremity of the same primary coil. So, there may be margin for reduction of the tag identification time, by simplifying the anti-collision protocol. In the same way, other RFID standards in HF band, such as the ISO/IEC-14443B variant, that can achieve higher bitrates, could also be tested, possibly yielding faster cycle response and higher maximum vehicle speeds.

The creation of a specialized RFID standard, working at a carrier frequency $1 / 4$ to $1 / 5$ of the current $13.56 \mathrm{MHz}$ standard, is perceived as opportune, as it would allow reader coils with perimeter up to $\lambda / 10=8.8 \mathrm{~m}$, capable of handling vehicle detection in larger volumes and at higher speeds, but still operating in the desired near-field mode. The $3.155-3.400 \mathrm{MHz}$ band has already been recommended for RFID, access control and automatic road tolling applications [17], and should better fit the DIWPT application.

\section{ACKNOWLEDGMENT}

This research was partially supported by grant SFRH/BD/52349/2013 from FCT, the Portuguese funding agency supporting science, technology and innovation, to whom the authors are deeply grateful.

\section{REFERENCES}

[1] S. Ahn et al., "Low frequency electromagnetic field reduction techniques for the On-Line Electric Vehicle (OLEV)," 2010 IEEE Int. Symp. Electromagn. Compat., pp. 625-630, 2010.

[2] S. Li and C. C. Mi, "Wireless Power Transfer for Electric Vehicle Applications," IEEE J. Emerg. Sel. Top. Power Electron., vol. 3, no. 1, pp. 4-17, 2015.

[3] J. M. Miller et al., "Demonstrating dynamic wireless charging of an electric vehicle: The benefit of electrochemical capacitor smoothing," IEEE Power Electron. Mag., no. March, pp. 12-24, 2014.

[4] G. R. Nagendra, L. Chen, G. A. Covic, and J. T. Boys, "Detection of EVs on IPT Highways," IEEE J. Emerg. Sel. Top. Power Electron., vol. 2, no. 3, pp. 584-597, Sep. 2014.

[5] B. S. Cook et al., "RFID-Based Sensors for Zero-Power Autonomous Wireless Sensor Networks," IEEE Sensors Journal., vol. 14, no. 8, pp. 2419-2431, Aug. 2014.

[6] European Committee for Standardization, "European standard NF EN 15194, Cycles - Electrically power assisted cycles - EPAC bicycles." European Committee for Standardization, 2009.

[7] L. A. L. Cardoso, M. C. Martinez, A. A. N. Meléndez, and J. L. Afonso, "Dynamic inductive power transfer lane design for e-bikes," in 2016 IEEE 19th International Conference on Intelligent Transportation Systems (ITSC), 2016, pp. 2307-2312.

[8] A. Kamineni, G. A. Covic, and J. T. Boys, "Interoperable EV detection for dynamic wireless charging with existing hardware and free resonance," IEEE PELS Work. Emerg. Technol. Wirel. Power, WoW 2016, pp. 169-173, 2016

[9] M. Budhia, J. T. Boys, G. A. Covic, and C. Y. Huang, "Development of a Single-Sided Flux Magnetic Coupler for Electric Vehicle IPT Charging Systems," IEEE Trans. Ind. Electron., vol. 60, no. 1, pp. 318-328, Jan. 2013.

[10] International Organization for Standardization; ISO/IEC 14443 1:2008(E), Identification cards - Contactless integrated circuit cards Proximity cards - Part 1: Physical characteristics, 2nd ed. 2008.

[11] International Organization for Standardization; ISO/IEC 14443 2:2001(E), Identification cards - Contactless integrated circuit(s) cards Proximity cards - Part 2: Radio frequency power and signal interface, 1st ed. 2001 .

[12] International Organization for Standardization; ISO/IEC 144433:2001(E), Identification cards - Contactless integrated circuit(s) cards Proximity cards - Part 3: Initialization and anticollision, 1st ed. 2001.

[13] NXP Semiconductors, MF1S50yyX/V1 MIFARE Classic EV1 $1 \mathrm{~K}$ Mainstream contactless smart card IC for fast and easy solution development, Rev. 3. 2014.

[14] I. Kirschenbaum and A. Wool, "How to Build a Low-cost, Extendedrange RFID Skimmer," in Proceedings of the 15th Conference on USENIX Security Symposium - Volume 15, 2006.

[15] K. Finkenzeller, F. Pfeiffer, and E. Biebl, "Range Extension of an ISO/IEC-14443 type A RFID System with Actively Emulating Load Modulation," in RFID SysTech 2011 7th European Workshop on Smart Objects: Systems, Technologies and Applications, 2011, pp. 1-10.

[16] NXP Semiconductors, MFRC522 Standard performance MIFARE and NTA G frontend, Rev. 3.9. 2016.

[17] CEPT Electronic Communications Committee, "ERC Recommendation 70-03, Relating to the use of Short Range Devices (SRD)," May, 2017., European Conference of Postal and Telecommunications Administrations. 